\title{
Kinematics and Energetics of the EUV Waves on 11 April 2013
}

\author{
Aarti Fulara ${ }^{1}$ (D) Ramesh Chandra ${ }^{1}$ (D) \\ P.F. Chen ${ }^{2,3}$ (D) Ivan Zhelyazkov ${ }^{4}$ (D) \\ A.K. Srivastava ${ }^{5}$ (D) Wahab $\operatorname{Uddin}^{6}$ (D)
}

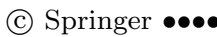

\begin{abstract}
In this study, we present the observations of extreme-ultraviolet (EUV) waves associated with an M6.5 flare on 2013 April 11. The event was observed by Solar Dynamics Observatory (SDO) in different EUV channels. The flare was also associated with a halo CME and type II radio bursts. We observed both fast and slow components of the EUV wave. The speed of the fast component, which is identified as a fast-mode MHD wave, varies in the range from 600 to $640 \mathrm{~km} \mathrm{~s}^{-1}$, whereas the speed of the slow-component is $\approx 140 \mathrm{~km} \mathrm{~s}^{-1}$. We observed an unusual phenomenon that, as the fast-component EUV wave passes through two successive magnetic quasi-separatrix layers (QSLs), two stationary wave fronts are formed locally. We propose that part of the outward-propagating fast-mode EUV wave is converted into slow-mode magnetohydrodynamic waves, which are trapped in local magnetic field structures, forming successive stationary fronts. Along the other direction, the fast-component EUV wave also creates oscillations in a coronal loop lying $\approx 225 \mathrm{Mm}$ away from the flare site. We have computed the energy of the EUV wave to be of the order of $10^{20} \mathrm{~J}$.
\end{abstract}

\footnotetext{
$凶$ Aarti Fulara

aarti.solarphysics@gmail.com

1 Department of Physics, DSB Campus, Kumaun University, Nainital 263001, India

2 School of Astronomy \& Space Science, Nanjing University, 163 Xianlin Ave, Nanjing 210023, China

3 Key Laboratory of Modern Astronomy \& Astrophysics (Nanjing University), Ministry of Education, Nanjing 210023, China

4 Faculty of Physics, Sofia University, 1164 Sofia, Bulgaria

5 Department of Physics, Indian Institute of Technology (BHU), Varanasi 221005, India

6 Aryabhatta Research Institute of Observational Sciences, Manora Peak, Nainital 263002, India
} 
Keywords: Waves, Magnetohydrodynamic; Waves, Propagation; Corona, Radio Emission

\section{Introduction}

Globally propagating disturbances traveling through the corona first came into existence by the observation made by the Extreme-Ultraviolet Imaging Telescope (EIT) (EIT: Delaboudinière et al., 1995) on board the Solar and Heliospheric Observatory (SOHO) (SOHO: Domingo, Fleck, and Poland, 1995), thus known as "EIT waves." Being clearly visible in various EUV wavelengths, they are also termed as EUV waves. EUV waves are characterized by bright and diffuse fronts which can sometimes travel the whole solar disk (Moses et al., 1997; Thompson et al., 1998). Their speeds range from a few tens $\mathrm{km} \mathrm{s}^{-1}$ up to more than $1000 \mathrm{~km} \mathrm{~s}^{-1}$ (Thompson and Myers, 2009; Nitta et al., 2013; Muhr et al., 2014, Long et al., 2017; Zheng et al., 2018).

Coronal mass ejections play an important role in the generation of EUV waves. Since CMEs are best detected above the solar limb and EUV waves are best seen on the disk, it is always difficult to clarify their association. A statistical analysis by Biesecker and Thompson (2002) revealed that all the clearly-identified EUV waves are associated with CMEs, though only $58 \%$ of all EUV waves, including faint events, are accompanied by CMEs. In contrast, only $20 \%$ of the CMEs have waves associated with them (Thompson and Myers, 2009). Muhr et al. (2014) did a more detailed study on the relationship between EUV waves and CMEs and found that $95 \%$ of 60 EUV waves in their sample are associated with CMEs. Nowadays, it is widely believed that EUV waves are intimately associated with CMEs, rather than solar flares. In particular, it was proposed that the CME frontal loop is cospatial with the slow-component of EUV waves, whereas the fast-component EUV wave corresponds to the piston-driven shock wave straddling over the CME (Chen, 2009). The cospatiality between the CME flank and the slow-component EUV wave was later confirmed by several authors (Chen, 2009 Attrill et al., 2009, Dai et al., 2010, Zhou and Liang, 2017). It is noted, however, that since EUV waves and CMEs are often observed in different fields of view, their association still requires more detailed studies.

Regarding the nature of EUV waves, several models have been proposed. Initially it was widely believed that EUV waves are fast-mode magnetohydrodynamic (MHD) waves (Thompson et al., 1998, Wang, 2000; Wu et al., 2001 Patsourakos and Vourlidas, 2009 Schmidt and Ofman, 2010), which was seconded by the observational features like wave reflection, refraction, and transmission (Gopalswamy et al., 2009, Kienreich et al., 2013, Veronig, Temmer, and Vršnak, 2008, Long et al., 2008). However, contrary to this, slower EUV waves, whose speeds are even smaller than the coronal sound speed, have also been observed. These waves cannot be explained by the fast-mode wave model, and have been proposed to be due to Joule heating (Delannée, Hochedez, and Aulanier, 2007), successive reconnection (Attrill et al., 2007), or slow-mode waves (Mei, Udo, and Lin, 2012). In addition, the existence of stationary fronts (Delannée, Hochedez, and Aulanier, 2007; Chandra et al., 2009) challenged the fast-mode 
wave model for all EUV waves. To reconcile all these discrepancies, Chen et al. (2002) and Chen, Fang, and Shibata (2005) proposed a hybrid wave theory, i.e. there are two types of EUV waves associated with one coronal mass ejection (CME) event, where the outer sharp wave front is a fast-mode MHD wave or shock wave and the inner diffuse front with a smaller speed is an apparent wave produced by successive stretching of magnetic field lines. Such a two-component EUV wave scenario was further supported by three-dimensional simulations (Cohen et al., 2009 Downs et al., 2012). With the high-cadence data, the coexistence of both the fast-mode wave and the slow-component EUV wave has been reported by many authors (Chen and Wu, 2011, Cheng et al., 2012 Asai et al., 2012 Kumar et al., 2013; Chandra et al., 2016 Zong and Dai, 2017; Chen, 2017). Recent reviews on EUV waves can be found in Warmuth (2007), WillsDavey and Attrill (2009), Warmuth (2010), Gallagher and Long (2011), Zhukov (2011), Liu and Ofman (2014), Warmuth (2015), and Chen (2016).

Delannée and Aulanier (1999) first reported the existence of stationary brightening in EUV images. They proposed that the stationary fronts are due to Joule heating of the electric currents generated near magnetic quasi-separatrix layers (QSLs) as the magnetic field lines are opening during a CME, and it was used as evidence to argue against the fast-mode wave model for EUV waves. Their work invoked Chen et al. (2002) to propose the magnetic fieldline stretching model for EIT waves, and this model can naturally explain why the slow-component EUV wave, i.e. the non-wave component stops at a magnetic QSL (Chen, Fang, and Shibata, 2005). On the other hand, Chandra et al. (2016) for the first time reported a different scenario in observations, i.e. in addition to a stationary front being the decelerating slow-component EUV wave, another stationary front is generated as the fast-component EUV wave passes through a magnetic QSL. The fast-component EUV wave continues its journey but with much reduced intensity. It was proposed by Chen et al. (2016) via MHD simulations that the new stationary front is a new-born slow-mode MHD wave, which is converted from the incident fast-mode EUV wave. Very recently, Zong and Dai (2017) and Chandra et al. (2018) found in observations that fast-mode EUV waves can indeed be converted into slow-mode waves when passing through helmet streamers, whose boundary corresponds to a magnetic QSL. More interestingly, Delannée, Hochedez, and Aulanier (2007) revealed multiple stationary fronts, which were visible in both EUV and $\mathrm{H} \alpha$ wavelengths. However, owing to the low cadence of the EIT telescope, they cannot tell whether the stationary fronts are produced by the opening of magnetic field lines or by the perturbation of the ambient coronal magnetic field while a magnetosonic wave passes through them.

The fast-component EUV waves are considered to be coronal shock waves. Shock waves signify the appearance of type II radio bursts. Type II radio bursts appear as strips of enhanced radio emission slowly drifting from high to low frequencies in the radio dynamic spectra. Biesecker and Thompson (2002) pointed out that a type II radio burst is a sufficient but not a necessary condition for an EUV wave. Recently, Long et al. (2017) did a statistical analysis of the events with EUV waves and type II radio bursts and found that only $40 \%$ of the wave events have type II bursts associated with them. Also, they reported that there is no clear relation between the velocity of the wave and the drift speed of the 
type II radio burst. Thus, this lack of clear relationship may be again attributed to the fact that EUV waves propagate in the low corona while radio bursts are related to the upper corona (Mann et al., 2003; Patsourakos and Vourlidas, 2009). Hence, their correlation is still controversial and needs more detailed studies.

Regarding the energetics of EUV waves, there are still controversies. Their energy varies from $10^{16}$ to $10^{24} \mathrm{~J}$. Therefore, studying the energetics of EUV waves, still awaits more efforts. A few papers addressing the energetics of EUV waves are summarised as follows: Ballai, Erdélyi, and Pintér (2005) computed the energy of an EUV wave based on the parameters of loop oscillations. The loop oscillations were produced by the EUV wave. Their calculations were based on typical coronal parameters such as the phase speed of the wave generated in the loop, sound speed, cusp speed (which is slow-mode speed in the external region) and density ratio inside and outside the loop. Their assumption was based on the fact that all the energy of the wave is transferred to the oscillating loop and this computed energy is the lower limit for the EUV wave. Based on the method proposed by Ballai, Erdélyi, and Pintér (2005), Ballai (2007) did a statistical analysis of 14 EUV wave events and obtained the energy of EUV waves in the range $10^{16}-10^{19} \mathrm{~J}$. Gilbert et al. (2008) studied a wave-filament interaction and computed the maximum total kinetic energy involved in the interaction. Their resulting energy is in the range of $\approx 10^{19}-10^{20} \mathrm{~J}$. Patsourakos and Vourlidas (2012) adopted a different approach to compute the total energy of EUV waves. They suggested that the energy of the EUV wave can be considered as the sum of three terms, i.e. the kinetic energy flux, the radiative loss flux, and the coronal thermal conduction flux. Their computed energy is of the order of $10^{22} \mathrm{~J}$. More recently, Long et al. (2015) also estimated the energy of the coronal waves using an approximation for shock waves which propagate in a region of variable density, and their energy of the EUV wave turns out to be $\approx 10^{24} \mathrm{~J}$.

In this paper we present the observations of an EUV wave event on 2013 April 11 originating from the active region NOAA AR 11719. Section 2 illustrates the observational data sets and the general overview of the event. In Section 3, we describe the kinematics and energetics of the EUV wave. The associated CME and type II radio bursts are described in Section 4. Finally in Section 5, we discuss and conclude our results.

\section{Observational Data Sets and General Overview of the Event}

The flare and the associated EUV wave on 2013 April 11 are well observed by the Atmospheric Imaging Assembly (AIA) (AIA: Lemen et al., 2012) on board the Solar Dynamics Observatory (SDO) (SDO: Pesnell, Thompson, and Chamberlin, 2012) satellite, which observes the full Sun at different wavelengths in EUV and UV with high spatial $\left(0.6^{\prime \prime}\right)$ and temporal $(12 \mathrm{~s})$ resolutions. For the present study, we use AIA $171 \AA, 193 \AA$, and $1600 \AA$ data. To see the chromospheric signatures of the flare and EUV waves, we use $\mathrm{H} \alpha$ data from the Global Oscillation Network Group (GONG) instruments. GONG observes the full Sun in $\mathrm{H} \alpha$ with a cadence of 1 min and a spatial resolution of $2^{\prime \prime}$. For the associated type II radio bursts and the associated CME, we use the data 
from the Hiraiso Radio Spectrograph (HiRAS) (HiRAS: Kondo et al., 1995) and the Large Angle and Spectrometric Coronagraph (LASCO) (LASCO: Brueckner et al., 1995) on board the SOHO satellite.

The active region NOAA AR 11719 appears near the east limb on 2013 April 7 and turns behind the west limb on 2013 April 17 . The flare starts at $\approx 06: 55$ UT on April 11 and is classified as M6.5 class according to the GOES X-ray flux. Before the flare onset, a sigmoidal structure is visible in AIA $94 \AA$ wavelength. The detailed study of this sigmoidal formation was done by Vemareddy and Mishra (2015) and Joshi et al. (2017). Figure 1 presents a multiwavelength view of the flare in different AIA channels and in GONG $\mathrm{H} \alpha$. The flare begins as two bright kernels, which gradually expand to form two reverse J-shaped ribbons. Figure 1. shows the peak phase of the flare at 07:10 UT in $\mathrm{H} \alpha$. The flare ribbons show separation from each other, which is typical for two-ribbon flares. The reverse J-shaped ribbon in the west indicates negative helicity in the active region as reported in several observations (Chandra et al., 2011, Schmieder, Aulanier, and Vršnak, 2015 Janvier, 2017). In Figure 1 d, we overplot the HMI magnetic field contours on the AIA $1600 \AA$ image. The structure of the flare ribbons as well as the existence of the sigmoid, both are consistent with negative helicity in the active region. The active region is located in the northern hemisphere which is dominated by negative helicity. Therefore, this active region follows the hemispheric rule (Ouyang et al., 2017).

\section{Results}

\subsection{Kinematics of the EUV Waves}

Figure 2 presents the propagation of the wave in the running difference images in AIA $193 \AA$. The difference images are created by subtracting the previous image as indicated in each image. The first trace of the wave is found at $\approx 07: 04 \mathrm{UT}$, and the EUV wave is quasi-circular in shape. It emanates from the active region and propagates mainly in the southeast direction away from the active region. As the wave progresses, its front becomes more and more diffuse. By 07:11 UT the wave almost reaches the east limb. As seen from Figure 2, the EUV wave is accompanied by dimmings in the wake. The dimmings are formed in the region enclosed between the flare site and the boundary of the EUV wave. Dimmings are often interpreted as regions of evacuation of coronal mass during a CME (Sterling and Hudson, 1997, Wang et al., 2002 Harra and Sterling, 2003 Zhukov and Auchère, 2004 Jin et al., 2009). However, there is distinction among the stronger core dimmings which are attributed to the footpoints of the flux rope (e.g. Sterling and Hudson, 1997; Webb et al., 2000), the fainter expanding dimmings, which are observed to trail behind the slow-component EUV wavefront (e.g. Delannée and Aulanier, 1999; Wills-Davey and Thompson, 1999), and the rarefaction in the wake of the fast-component EUV wave (e.g. Muhr et al., 2011, Lulić et al., 2013). What we see in Figure 2 are mainly the expanding dimmings and the rarefaction as indicated by the time-distance 
diagrams later shown in Figures 3 and 5 , whereas the core dimmings are localized at the boundaries of the source active region.

In order to study the kinematics of the observed EUV waves, after examining their propagation along several artificial slices in different directions from the flare site, we select two representative slices, where the propagation of EUV waves is clearly visible. We label them Slice 1 and Slice 2 , respectively. Let us first discuss about Slice 1. This slice extends toward the east direction (marked by the black curved line in Figure 3 a). The corresponding time-distance diagram of the base-difference AIA $193 \AA$ intensity is shown in Figure 3b. A prominent feature in Figure 3 is that a fast-moving wave propagates toward the east direction. The calculated speed is $\approx 640 \mathrm{~km} \mathrm{~s}^{-1}$, which is several times larger than the typical sound speed in the corona and is typical for the fast-component EUV waves (Chen, 2016). That is the reason why we call it a fast-mode EUV wave. Although the slow-component EUV wave is not as discernable here as in Chen and $\mathrm{Wu}$ (2011) and Kumar et al. (2013), one can still see two patchy brightenings as demonstrated by Guo, Ding, and Chen (2015). The transiting of the sequential brightenings forms a wavelike pattern, which can also be explained by the magnetic fieldline stretching model. Moreover, it is seen that these sequential patchy brightenings bound the expanding dimmings, as expected from the magnetic fieldline stretching model. Another interesting feature in Figure 3 is that when the fast-mode EUV wave propagates outward, two stationary brightenings are generated, which are marked as $\mathrm{SB}_{1}$ and $\mathrm{SB}_{2}$, respectively. The lifetimes of these stationary brightenings are tens of minutes.

In Figure 4 we compare the locations of the two stationary brightenings with the coronal magnetic field, which is extrapolated from the photospheric magnetogram with the potential field source surface (PFSS) model. It reveals that the two stationary brightenings along Slice 1 are both located at magnetic QSLs, where magnetic connectivity changes abruptly.

We also trace the propagation of the EUV waves along Slice 2, which is along the southeast direction as indicated in Figure 5 a. The corresponding timedistance diagram of the base-difference AIA $193 \AA$ intensity is presented in Figure 5b. Along Slice 2, we find the co-existence of two EUV waves, i.e. a fast component and a slow component. According to the magnetic fieldline stretching model, the fast-component EUV wave is a fast-mode MHD wave or shock wave, whereas the slow-component EUV wave is an apparent motion generated by the successive stretching of magnetic field lines pushed by an erupting flux rope (Chen et al., 2002). The speed of the fast-mode wave is $\approx 600 \mathrm{~km} \mathrm{~s}^{-1}$, which is again several times higher than the coronal sound speed and this speed is similar to the speed of fast-mode wave along Slice 1 . The speed of the slow-component EUV wave is $\approx 140 \mathrm{~km} \mathrm{~s}^{-1}$, which is about 4.3 times smaller than that of the fast-component EUV wave.

\subsection{Energetics of the EUV Wave}

Figure 6 shows an AIA $171 \AA$ image at 07:20 UT. We can see a coronal loop $\mathrm{L}_{1}$ indicated by the white arrow. As the EUV wave propagates along Slice 2, it encounters the loop system $\mathrm{L}_{1}$. The $\mathrm{L}_{1}$ loop starts to oscillate along the Slice 
Table 1. Observational parameters derived from the AIA observations.

\begin{tabular}{ll}
\hline Input parameters & Value \\
\hline Length of the loop $(L)$ & $198 \mathrm{Mm}$ \\
Coronal magnetic field $(B)$ & $8.23 \mathrm{G}(\mathrm{Guo}$ et al., 2015) \\
Number density $\left(n_{i}\right)$ & $5.1 \times 10^{8} \mathrm{~cm}^{-3}(\mathrm{GuO}$ et al., 2015 \\
Radius of the loop $(R)$ & $4.3 \mathrm{Mm}$ \\
Temperature $(T)$ & $0.65 \mathrm{MK}(\mathrm{Guo}$ et al., 2015$)$ \\
Mass density inside the loop $\left(\rho_{\mathrm{i}}\right)$ & $8.16 \times 10^{-13} \mathrm{~kg} \mathrm{~m}^{-3}$ \\
Mass density outside the loop $\left(\rho_{\mathrm{e}}\right)$ & $6.27 \times 10^{-13} \mathrm{~kg} \mathrm{~m}^{-3}$ \\
Maximum deflection of the loop $\left(x_{\max }\right)$ & $1.9 \mathrm{Mm}$ \\
Intermediate deflection of the loop $\left(x_{1}\right)$ & $1.2 \mathrm{Mm}$ \\
Time at which maximum deflection occurs $\left(t_{\mathrm{max}}\right)$ & $540 \mathrm{~s}$ \\
Time at which intermediate deflection occurs $\left(t_{1}\right)$ & $1080 \mathrm{~s}$ \\
Sound speed outside the loop $\left(c_{\mathrm{se}}\right)$ & $94.6 \mathrm{~km} \mathrm{~s}^{-1}$ \\
Alfvén speed inside the loop $\left(v_{\mathrm{Ai}}\right)$ & $791.6 \mathrm{~km} \mathrm{~s}^{-1}$ \\
Alfvén speed outside the loop $\left(v_{\mathrm{Ae}}\right)$ & $904.3 \mathrm{~km} \mathrm{~s}^{-1}$ \\
Phase speed in the loop $\left(v_{\mathrm{ph}}\right)$ & $841.6 \mathrm{~km} \mathrm{~s}^{-1}$ \\
Cusp speed outside the loop $\left(c_{\mathrm{Te}}\right)$ & $94.1 \mathrm{~km} \mathrm{~s}^{-1}$ \\
Wave number $(k)$ & $1.5 \times 10^{-8} \mathrm{~m}^{-1}$ \\
\hline
\end{tabular}

2 direction. We observe strong and clear oscillations of this loop. This loop system is $\approx 225 \mathrm{Mm}$ away from the flare site. Guo et al. (2015) also studied the oscillations of this particular loop. For the computation of the electron density inside the coronal loop, they calculated the background subtracted EUV fluxes in the six AIA wavelengths. Using these they derived the average electron density inside the loop to be $5.1 \pm 0.8 \times 10^{8} \mathrm{~cm}^{-3}$ and the temperature to be $0.65 \pm 0.06 \mathrm{MK}$. Via coronal seismology they derived the magnetic field strength, which is $B_{\mathrm{i}}=8.2 \mathrm{G}$. We created a time-distance plot using a series of $171 \AA$ images. Figure 7 a shows the position of the slice along which the $171 \AA$ intensity distribution is extracted. Figure $7 \mathrm{p}$ shows the time-slice diagram illustrating the loop oscillations. As seen from the figure, the first shift of the loop is in a direction away from the active region. Initially, the amplitude of the oscillations is large, but then decays. Using this time-distance diagram, we create an intensity-time plot for the oscillations which is shown in Figure 8 From the plot, we can see that the the maximum deflection of the loop is $1.9 \times 10^{4} \mathrm{~km}$. We compute the period of the loop oscillations using the wavelet analysis. Wavelet analysis helps us study the time-dependent period in the observed light-curves Torrence and Compo (1998). The period of the loop oscillations revealed from the wavelet analysis is $541 \mathrm{~s}$. The power spectrum of the wavelet analysis is shown in Figure 8 . Along with other computed observational parameters such as the length of the loop, magnetic field, temperature, and the radius of the loop, we also calculate the maximum and intermediate deflections of the loop and note down the respective times at which these deflections take place. 
As mentioned in Section 1 , the studies on the energy computation of the EUV waves have been done by many authors. But the difference between ours and the previous studies is that, in earlier studies (Ballai, Erdélyi, and Pintér, 2005. Ballai, 2007, Patsourakos and Vourlidas (2012)) typical coronal values of the sound speed, cusp speed, phase speed, Alfvén speed, temperature, and coronal density were used. To enhance our understanding on the energetics of the EUV waves, we derive these values directly from the observations. Note that some parameters are adopted from Guo et al. (2015), which are also based on observations. Thus, our study gives a more realistic energy value. The observational parameters used for the energy calculation are listed in Table 1.

We calculate the energy of the EUV wave around the coronal loop $\mathrm{L}_{1}$ using the following expression given by Ballai, Erdélyi, and Pintér (2005),

$$
\begin{gathered}
E=\frac{\pi L\left(\rho_{\mathrm{i}} R^{2}+\rho_{\mathrm{e}} / \lambda_{\mathrm{e}}^{2}\right)}{2}\left(\frac{x_{\max }-x_{1}}{t_{\max }-t_{1}}\right)^{2}, \\
\lambda_{\mathrm{e}}^{2}=\frac{\left(c_{\mathrm{se}}^{2}-v_{\mathrm{ph}}^{2}\right)\left(v_{\mathrm{Ae}}^{2}-v_{\mathrm{ph}}^{2}\right)}{\left(c_{\mathrm{se}}^{2}+v_{\mathrm{Ae}}^{2}\right)\left(c_{\mathrm{Te}}^{2}-v_{\mathrm{ph}}^{2}\right)} k^{2},
\end{gathered}
$$

where $\lambda_{\mathrm{e}}^{-1}$ is the decay length of perturbations outside the loop.

The basic parameters (sound and Alfvén speeds) used in Equation 2 are calculated from the pressure balance equation with the following input data investigated by Guo et al. (2015) and by us: $\rho_{\mathrm{i}}=8.53 \times 10^{-13} \mathrm{~kg} \mathrm{~m}^{-3}, \rho_{\mathrm{e}}=$ $6.56 \times 10^{-13} \mathrm{~kg} \mathrm{~m}^{-3}, T=0.65 \mathrm{MK}$, and $B_{\mathrm{i}}=8.2 \mathrm{G}$, to get:

$$
c_{\mathrm{se}}=94.6 \mathrm{~km} \mathrm{~s}^{-1}
$$

along with

$$
v_{\mathrm{Ai}}=791.6 \mathrm{kms}^{-1} \quad \text { and } \quad v_{\mathrm{Ae}}=904.3 \mathrm{~km} \mathrm{~s}^{-1} .
$$

Hence, the tube/cusp speed outside the loop is

$$
c_{\mathrm{Te}}=\frac{c_{\mathrm{se}} v_{\mathrm{Ae}}}{\sqrt{c_{\mathrm{se}}^{2}+v_{\mathrm{Ae}}^{2}}}=94.1 \mathrm{~km} \mathrm{~s}^{-1} .
$$

Since the oscillations are due to kink-mode waves, and considering that the kink mode is essentially non-dispersive, the phase velocity is equal to the kink speed. Thus, wave phase speed in Equation 2 is

$$
v_{\mathrm{ph}}=\sqrt{\frac{2}{1+\rho_{\mathrm{e}} / \rho_{\mathrm{i}}}} v_{\mathrm{Ai}} .
$$

Bearing in mind that the density contrast $\rho_{\mathrm{e}} / \rho_{\mathrm{i}}$ obtained by Guo et al. (2015) is equal to 0.769 , and the Alfvén speed inside the loop is of $791.6 \mathrm{~km} \mathrm{~s}^{-1}$, then the phase speed equals $841.6 \mathrm{~km} \mathrm{~s}^{-1}$.

With these speeds and wavenumber $k=1.5 \times 10^{-8} \mathrm{~m}^{-1}$, the value of $\lambda_{\mathrm{e}}^{2}$ calculated from Equation 1 is equal to $0.298 \times 10^{-16} \mathrm{~m}^{-2}$. Taking the magnitudes 
of the length and radius of the loop, $L$ and $R$, as well as the maximum and intermediate deflections of the loop, $x_{\max }$ and $x_{1}$, along with the corresponding times $t_{\max }$ and $t_{1}$, we obtain, from Equation 1 the minimum energy of the EUV wave transferred to the loop is $8.88 \times 10^{18} \mathrm{~J}$. Assuming that the wave is isotropic, we can estimate the total energy of the EUV wave by multiplying this minimum energy value, with a factor $R_{\mathrm{a}}$, where, $R_{\mathrm{a}}=2 \pi d^{2} / A$ is the area ratio between the EUV wave dome and the coronal loop $\mathrm{L}_{1}, d$ is the distance of the loop from the flare site, $A$ is the square of the distance between the foot points of the coronal loop (which is $145^{\prime \prime}$ ). The value of $R_{a}$ is 30 . Thus, the total energy of the EUV wave is $2.7 \times 10^{20} \mathrm{~J}$.

\section{CME and Radio Observations}

The M6.5 flare is associated with a full-halo CME. Figure 9 shows the $\mathrm{C} 2$ and C3 images observed by the LASCO coronagraph. At first, the CME appears in the $\mathrm{C} 2$ field of view at 07:36 UT, marked by white arrows as shown in Figure 9. It reaches the C3 field-of-view around 09:06 UT. The speed of the CME from the linear fit is estimated to be $861.5 \mathrm{~km} \mathrm{~s}^{-1}$. The acceleration of the CME from the quadratic fit is estimated to be $-8.07 \mathrm{~m} \mathrm{~s}^{-2}$.

Figure 10 shows the radio dynamic spectrum observed by the HiRAS Radio Spectrograph on 2013 April 11. Whereas a type II radio burst is clearly discernable, a type III radio burst is fairly weak. The type III burst is observed at 06:58-06:59 UT. However, the derivative of the GOES soft X-ray light curve, representing the hard $\mathrm{X}$-ray emission or the magnetic reconnection rate, peaks at 07:10 UT. It seems that the type III radio burst occurs when the derivative of the GOES soft X-ray flux starts to increase. The type II burst is observed to commence at 07:03 UT, which is 5 minutes later than the onset time of the fastmode EUV wave. A distinct feature of the type II radio burst is that both the fundamental and harmonic components are so wide in frequency that the two components merge together, and are not separate as in most events. In order to calculate the propagation speed of the shock wave, we trace the evolution of the lower branch of the harmonic component, which is $92 \mathrm{MHz}$ at 07:03 UT and $50 \mathrm{MHz}$ at 07:09 UT. To derive the shock wave speed from the radio dynamic spectrum, we need to assume a density model for the corona above the source region, which is unknown. If we take the one-fold Newkirk coronal density model Newkirk (1961), which is similar to that derived by Zucca et al. (2014), the resulting shock speed would be too much smaller than the CME speed, which is probably not reasonable. According to Newkirk (1961), the twofold model is suitable for average active regions, we therefore take the two-fold Newkirk density model, the corresponding heights of the type II radio source region are $0.73 \mathrm{R}_{\odot}$ and $1.19 \mathrm{R}_{\odot}$ above the solar surface, and its estimated radial propagating speed is about $896 \mathrm{~km} \mathrm{~s}^{-1}$. 


\section{Summary and Discussion}

In this paper, we present the kinematics of an EUV wave event, which is associated with an M6.5-class flare in AR NOAA 11719 and a halo CME on 2013 April 11. Our main results are summarized as follows:

- Along the east direction away from the source region, only the fast - component EUV wave with a speed of $\approx 640 \mathrm{~km} \mathrm{~s}^{-1}$ can be seen, which corresponds to a fast-mode wave or shock wave. Along the southeast direction, two EUV waves are visible, where the fast-mode wave travels with a speed of $\approx 600 \mathrm{~km} \mathrm{~s}^{-1}$ and the slow-component EUV wave travels with a speed of $\approx 140 \mathrm{~km} \mathrm{~s}^{-1}$. The slow-component EUV wave is 4.3 times slower than the fast-mode wave.

- When the fast-mode EUV wave propagates eastward, two stationary bright fronts are left behind. The locations of the two stationary fronts correspond to magnetic QSLs, where magnetic field changes its connectivity drastically.

- Based on the observational parameters of the oscillating loop system $\mathrm{L}_{1}$, we estimated the total energy of the EUV wave, which is $\approx 2.7 \times 10^{20} \mathrm{~J}$.

- Based on the type II burst in the radio dynamic spectrum, we derived the altitude of the CME-driven shock to be $0.73 \mathrm{R}_{\odot}$ at 07:03 $\mathrm{UT}$ and $1.19 \mathrm{R}_{\odot}$ at 07:09 UT above the solar surface.

After being discovered in 1997 (Thompson et al., 1998), EUV waves were initially thought to be fast-mode MHD waves or shock waves in the solar corona. However, the extremely low speeds (smaller than the coronal sound speed) in some events, and in particular, the discovery of stationary EUV wave front at magnetic QSLs (Delannée and Aulanier, 1999), invoked Chen et al. (2002) to propose a two-wave scenario, i.e. when a CME happens, there should exist two types of EUV waves, a fast-component EUV wave (which is a fast-mode wave or shock wave and corresponds to the coronal counterpart of the chromospheric Moreton wave) and a slow-component EUV wave (which is an apparent propagation produced by successive stretching of the closed magnetic field lines overlying the CME). The MHD numerical simulation performed by Chen, Fang, and Shibata (2005) indeed showed that the slow-component EUV wave stops at magnetic QSLs, a natural result of the magnetic fieldline stretching model. However, Delannée, Hochedez, and Aulanier (2007) found that even the Moreton wave can also generate a stationary front in $\mathrm{H} \alpha$. It implies that there should be two different mechanisms for the formation of stationary fronts. Although Delannée, Hochedez, and Aulanier (2007) already presented multiple stationary EUV wave fronts, they could not pin down the formation process owing to the low cadence of the EIT telescope. With the high cadence data observed by SDO/AIA, Chandra et al. (2016) revealed that when the fast-component EUV wave passes through a magnetic QSL, a stationary EUV wave front is generated. In order to explain the formation of this new kind of stationary wave front, Chen et al. 
(2016) proposed that before reaching a magnetic QSL, part of the fast-mode MHD wave in the corona is converted to a slow-mode MHD wave at the location where the Alfvén speed is equal to the sound speed. Since a slow-mode wave can only propagate along the magnetic field line until it decays at the footpoint of the field line, it is seen to be a stationary front as viewed from above. Since the slow-mode wave will finally be dissipated in the chromosphere, this model can also explain the stationary $\mathrm{H} \alpha$ front discovered by Delannée, Hochedez, and Aulanier (2007). Such a model was confirmed by later observations (Zong and Dai, 2017; Chandra et al., 2018). However, these observations displayed only a single stationary EUV wave front. In the real corona, a fast-mode EUV wave might pass through several magnetic QSLs, and therefore, it is expected to see the formation of a series of stationary EUV waves. In this paper, we showed that in the 2013 April 11 event, as the fast-mode EUV wave propagates outward to the east, two stationary EUV wave fronts are produced. The locations of the stationary fronts are exactly near magnetic QSLs. The schematic representation of the observations and the formation of stationary fronts is shown in Figure 11.

Although Delannée, Hochedez, and Aulanier (2007) already showed the observation of multiple stationary EUV wave fronts, the low-cadence data did not allow them to pin down how these stationary fronts are formed. Besides, we proposed an alternative explanation for the formation of these stationary fronts. Since they are formed by the CME-driven shock wave, we tend to think that they are not related to the magnetic rearrangement, which happens much behind the shock wave.

It should also be noted that although the MHD simulations performed by Chen et al. (2002) displayed two types of EUV waves as a CME erupts, it does not mean that one can always see two different EUV waves along any direction. According to the magnetic fieldline stretching model, the slow-component EUV wave is generated by the stretching of the magnetic field lines straddling over the erupting flux rope. Therefore, the slow-component EUV wave appears only in the regions whose magnetic field lines straddle over the erupting flux rope. In contrast, the fast-component EUV wave, which is a CME-driven shock wave, would be more circular. As revealed in this paper, we can see only the fastcomponent EUV wave along the eastward direction (Slice 1) and two EUV waves are discernable along the southeast direction, which implies that the large-scale coronal magnetic field overlying the erupting flux rope is mainly along the southeast direction. Such an inference is consistent with the observational fact that the two ribbons of the associated flare, as indicated by Figure 1 are separated in the southeast-northwest direction. Besides, according to the magnetic fieldline stretching model, the slow-component EUV wave would be $\approx 3$ times slower than the fast-component EUV wave if the magnetic field lines are concentric semicircles. In this paper, the slow-component EUV wave is $\approx 4.3$ times slower than the fast-component EUV wave, implying that the coronal magnetic field lines are more elongated in the solar radial direction (Chen, Fang, and Shibata, 2005).

According to the magnetic fieldline stretching model, the fast-component EUV wave and the type II burst source region represent different parts of 
the same CME piston-driven shock wave. Therefore, the locations of the fastcomponent EUV wave and the type II radio bursts can provide the shape information of the CME-driven shock wave when it can not be directly imaged as in Ma et al. (2011). With the Newkirk 2-fold density model, the radio burst is at the altitude of $0.73 \mathrm{R}_{\odot}$ above the solar surface at 07:03 UT. At this moment, the fast-component EUV wave is $350^{\prime \prime}$ (i.e. $0.37 \mathrm{R}_{\odot}$ ) away from the flare site according to Figure 3 implying that the shock wave is relatively elongated in the solar radial direction. It is noted in passing that we found that the fastcomponent EUV wave appears 5 minutes before the onset of the type II radio burst. This result is of significance in clarifying the debate about whether the commencement of type II radio burst is indicative of the formation of a shock wave. Our observation implies that only when the shock is strong enough, e.g. a supercritical shock (Benz and Thejappa, 1988), can a type II radio burst be excited ( $\mathrm{Su}$ et al., 2016).

Similar to the study of Ballai, Erdélyi, and Pintér (2005) and Ballai (2007), we observed loop oscillations as the fast-component EUV wave impinges the coronal loop. To estimate the total energy of the EUV wave, we adopted the computation method proposed by the above authors. Different from Ballai, Erdélyi, and Pintér (2005) who used typical coronal physical parameters in their calculation, we derived the input physical parameters using the SDO/AIA data. For the EUV wave event studied in this paper, the total energy is estimated to be $2.7 \times 10^{20} \mathrm{~J}$.

Acknowledgments We would like to thank the referee for the useful comments and suggestions that helped us to improve the manuscript. We also acknowledge the use of SDO and GONG data. PFC was supported by the Chinese grants NSFC 11533005, U1731241 and Jiangsu 333 Project (No. BRA2017359). AF acknowledges the support from the ISRO/RESPOND project. The work of IZh and RC was supported by the Bulgarian Science Fund under IndoBulgarian bilateral project DNTS/INDIA 01/7.

Disclosure of Potential Conflicts of Interest The authors declare that they have no conflicts of interest.

\section{References}

Asai, A., Ishii, T.T., Isobe, H., Kitai, R., Ichimoto, K., UeNo, S., Nagata, S., Morita, S., Nishida, K., Shiota, D., Oi, A., Akioka, M., Shibata, K.: 2012, First Simultaneous Observation of an $\mathrm{H} \alpha$ Moreton Wave, EUV Wave, and Filament/Prominence Oscillations. Astrophys. J. Lett. 745, L18. DOI ADS

Attrill, G.D.R., Harra, L.K., van Driel-Gesztelyi, L., Démoulin, P.: 2007, Coronal "Wave": Magnetic Footprint of a Coronal Mass Ejection? Astrophys. J. Lett. 656, L101. DOI ADS

Attrill, G.D.R., Engell, A.J., Wills-Davey, M.J., Grigis, P., Testa, P.: 2009, Hinode/XRT and STEREO Observations of a Diffuse Coronal "Wave"-Coronal Mass Ejection-Dimming Event. Astrophys. J. 704, 1296. DOI ADS

Ballai, I.: 2007, Global Coronal Seismology. Solar Phys. 246, 177. DOI ADS

Ballai, I., Erdélyi, R., Pintér, B.: 2005, On the Nature of Coronal EIT Waves. Astrophys. J. Lett. 633, L145. DOI ADS

Benz, A.O., Thejappa, G.: 1988, Radio emission of coronal shock waves. Astron. Astrophys. 202, 267. ADS 
Biesecker, D.A., Thompson, B.J.: 2002, Can EIT Waves be used to Predict Halo CME Properties? In: American Astronomical Society Meeting Abstracts \#200, Bulletin of the American Astronomical Society 34, 695.

Brueckner, G.E., Howard, R.A., Koomen, M.J., Korendyke, C.M., Michels, D.J., Moses, J.D., Socker, D.G., Dere, K.P., Lamy, P.L., Llebaria, A., Bout, M.V., Schwenn, R., Simnett, G.M., Bedford, D.K., Eyles, C.J.: 1995, The Large Angle Spectroscopic Coronagraph (LASCO). Solar Phys. 162, 357. DOI ADS

Chandra, R., Schmieder, B., Aulanier, G., Malherbe, J.M.: 2009, Evidence of Magnetic Helicity in Emerging Flux and Associated Flare. Solar Phys. 258, 53. DOI. ADS

Chandra, R., Schmieder, B., Mandrini, C.H., Démoulin, P., Pariat, E., Török, T., Uddin, W.: 2011, Homologous Flares and Magnetic Field Topology in Active Region NOAA 10501 on 20 November 2003. Solar Phys. 269, 83. DOI ADS.

Chandra, R., Chen, P.F., Fulara, A., Srivastava, A.K., Uddin, W.: 2016, Peculiar Stationary EUV Wave Fronts in the Eruption on 2011 May 11. Astrophys. J. 822, 106. DOI ADS

Chandra, R., Chen, P.F., Joshi, R., Joshi, B., Schmieder, B.: 2018, Observations of Two Successive EUV Waves and Their Mode Conversion. Astrophys. J. 863, 101. DOI. ADS

Chen, P.: 2017, The continued debate on solar coronal EUV waves. Science China Physics, Mechanics, and Astronomy 60, 29631. DOI ADS

Chen, P.F.: 2009, The Relation Between EIT Waves and Coronal Mass Ejections. Astrophys. J. Lett. 698, L112. DOI. ADS

Chen, P.F.: 2016, Global Coronal Waves. Washington DC American Geophysical Union Geophysical Monograph Series 216, 381. DOI ADS

Chen, P.F., Wu, Y.: 2011, First Evidence of Coexisting EIT Wave and Coronal Moreton Wave from SDO/AIA Observations. Astrophys. J. Lett. 732, L20. DOI ADS

Chen, P.F., Fang, C., Shibata, K.: 2005, A Full View of EIT Waves. Astrophys. J. 622, 1202. DOI. ADS

Chen, P.F., Wu, S.T., Shibata, K., Fang, C.: 2002, Evidence of EIT and Moreton Waves in Numerical Simulations. Astrophys. J. Lett. 572, L99. DOI ADS

Chen, P.F., Fang, C., Chandra, R., Srivastava, A.K.: 2016, Can a Fast-Mode EUV Wave Generate a Stationary Front? Solar Phys. 291, 3195. DOI. ADS.

Cheng, X., Zhang, J., Olmedo, O., Vourlidas, A., Ding, M.D., Liu, Y.: 2012, Investigation of the Formation and Separation of an Extreme-ultraviolet Wave from the Expansion of a Coronal Mass Ejection. Astrophys. J. Lett. 745, L5. DOI ADS.

Cohen, O., Attrill, G.D.R., Manchester, W.B. IV, Wills-Davey, M.J.: 2009, Numerical Simulation of an EUV Coronal Wave Based on the 2009 February 13 CME Event Observed by STEREO. Astrophys. J. 705, 587. DOI. ADS

Dai, Y., Auchère, F., Vial, J.-C., Tang, Y.H., Zong, W.G.: 2010, Large-scale ExtremeUltraviolet Disturbances Associated with a Limb Coronal Mass Ejection. Astrophys. J. 708, 913. DOI ADS

Delaboudinière, J.-P., Artzner, G.E., Brunaud, J., Gabriel, A.H., Hochedez, J.F., Millier, F., Song, X.Y., Au, B., Dere, K.P., Howard, R.A., Kreplin, R., Michels, D.J., Moses, J.D., Defise, J.M., Jamar, C., Rochus, P., Chauvineau, J.P., Marioge, J.P., Catura, R.C., Lemen, J.R., Shing, L., Stern, R.A., Gurman, J.B., Neupert, W.M., Maucherat, A., Clette, F., Cugnon, P., van Dessel, E.L.: 1995, EIT: Extreme-Ultraviolet Imaging Telescope for the SOHO Mission. Solar Phys. 162, 291. DOI. ADS.

Delannée, C., Aulanier, G.: 1999, Cme Associated with Transequatorial Loops and a Bald Patch Flare. Solar Phys. 190, 107. DOI ADS.

Delannée, C., Hochedez, J.-F., Aulanier, G.: 2007, Stationary parts of an EIT and Moreton wave: a topological model. Astron. Astrophys. 465, 603. DOI. ADS

Domingo, V., Fleck, B., Poland, A.I.: 1995, The SOHO Mission: an Overview. Solar Phys. 162, 1. DOI ADS

Downs, C., Roussev, I.I., van der Holst, B., Lugaz, N., Sokolov, I.V.: 2012, Understanding SDO/AIA Observations of the 2010 June 13 EUV Wave Event: Direct Insight from a Global Thermodynamic MHD Simulation. Astrophys. J. 750, 134. DOI

Gallagher, P.T., Long, D.M.: 2011, Large-scale Bright Fronts in the Solar Corona: A Review of "EIT waves". Space Sci. Rev. 158, 365. DOI ADS

Gilbert, H.R., Daou, A.G., Young, D., Tripathi, D., Alexander, D.: 2008, The FilamentMoreton Wave Interaction of 2006 December 6. Astrophys. J. 685, 629. DOI ADS

Gopalswamy, N., Yashiro, S., Temmer, M., Davila, J., Thompson, W.T., Jones, S., McAteer, R.T.J., Wuelser, J.-P., Freeland, S., Howard, R.A.: 2009, EUV Wave Reflection from a Coronal Hole. Astrophys. J. Lett. 691, L123. DOI ADS 
Guo, Y., Ding, M.D., Chen, P.F.: 2015, Slow Patchy Extreme-ultraviolet Propagating Fronts Associated with Fast Coronal Magneto-acoustic Waves in Solar Eruptions. Astrophys. J. Supplement Series 219, 36. DOI ADS

Guo, Y., Erdélyi, R., Srivastava, A.K., Hao, Q., Cheng, X., Chen, P.F., Ding, M.D., Dwivedi, B.N.: 2015, Magnetohydrodynamic Seismology of a Coronal Loop System by the First Two Modes of Standing Kink Waves. Astrophys. J. 799, 151. DOI ADS

Harra, L.K., Sterling, A.C.: 2003, Imaging and Spectroscopic Investigations of a Solar Coronal Wave: Properties of the Wave Front and Associated Erupting Material. Astrophys. J. 587, 429. DOI ADS

Janvier, M.: 2017, Three-dimensional magnetic reconnection and its application to solar flares. Journal of Plasma Physics 83, 535830101. DOI ADS

Jin, M., Ding, M.D., Chen, P.F., Fang, C., Imada, S.: 2009, Coronal Mass Ejection Induced Outflows Observed with Hinode/EIS. Astrophys. J. 702, 27. DOI. ADS

Joshi, B., Kushwaha, U., Veronig, A.M., Dhara, S.K., Shanmugaraju, A., Moon, Y.-J.: 2017, Formation and Eruption of a Flux Rope from the Sigmoid Active Region NOAA 11719 and Associated M6.5 Flare: A Multi-wavelength Study. Astrophys. J. 834, 42. DOI. ADS

Kienreich, I.W., Muhr, N., Veronig, A.M., Berghmans, D., De Groof, A., Temmer, M., Vršnak, B., Seaton, D.B.: 2013, Solar TErrestrial Relations Observatory-A (STEREO-A) and PRoject for On-Board Autonomy 2 (PROBA2) Quadrature Observations of Reflections of Three EUV Waves from a Coronal Hole. Solar Phys. 286, 201. DOI ADS.

Kondo, T., Isobe, T., Igi, S., Watari, S., Tokimura, M.: 1995, The Hiraiso Radio Spectrograph (HiRAS) for monitoring solar radio bursts. J. Commun. Res. Lab., Vol. 42, No. 1, p. 111 - 119 42, 111. ADS

Kumar, P., Cho, K.-S., Chen, P.F., Bong, S.-C., Park, S.-H.: 2013, Multiwavelength Study of a Solar Eruption from AR NOAA 11112: II. Large-Scale Coronal Wave and Loop Oscillation. Solar Phys. 282, 523. DOI

Lemen, J.R., Title, A.M., Akin, D.J., Boerner, P.F., Chou, C., Drake, J.F., Duncan, D.W., Edwards, C.G., Friedlaender, F.M., Heyman, G.F., Hurlburt, N.E., Katz, N.L., Kushner, G.D., Levay, M., Lindgren, R.W., Mathur, D.P., McFeaters, E.L., Mitchell, S., Rehse, R.A., Schrijver, C.J., Springer, L.A., Stern, R.A., Tarbell, T.D., Wuelser, J.-P., Wolfson, C.J., Yanari, C., Bookbinder, J.A., Cheimets, P.N., Caldwell, D., Deluca, E.E., Gates, R., Golub, L., Park, S., Podgorski, W.A., Bush, R.I., Scherrer, P.H., Gummin, M.A., Smith, P., Auker, G., Jerram, P., Pool, P., Soufli, R., Windt, D.L., Beardsley, S., Clapp, M., Lang, J., Waltham, N.: 2012, The Atmospheric Imaging Assembly (AIA) on the Solar Dynamics Observatory (SDO). Solar Phys. 275, 17. DOI ADS

Liu, W., Ofman, L.: 2014, Advances in Observing Various Coronal EUV Waves in the SDO Era and Their Seismological Applications (Invited Review). Solar Phys. 289, 3233. DOI ADS

Long, D.M., Gallagher, P.T., McAteer, R.T.J., Bloomfield, D.S.: 2008, The Kinematics of a Globally Propagating Disturbance in the Solar Corona. Astrophys. J. Lett. 680, L81. DOI] ADS

Long, D.M., Baker, D., Williams, D.R., Carley, E.P., Gallagher, P.T., Zucca, P.: 2015, The Energetics of a Global Shock Wave in the Low Solar Corona. Astrophys. J. 799, 224. DOI ADS

Long, D.M., Bloomfield, D.S., Chen, P.F., Downs, C., Gallagher, P.T., Kwon, R.-Y., Vanninathan, K., Veronig, A.M., Vourlidas, A., Vršnak, B., Warmuth, A., Žic, T.: 2017, Understanding the Physical Nature of Coronal "EIT Waves". Solar Phys. 292, 7. DOI ADS

Lulić, S., Vršnak, B., Žic, T., Kienreich, I.W., Muhr, N., Temmer, M., Veronig, A.M.: 2013, Formation of Coronal Shock Waves. Solar Phys. 286, 509. DOI ADS

Ma, S., Raymond, J.C., Golub, L., Lin, J., Chen, H., Grigis, P., Testa, P., Long, D.: 2011, Observations and Interpretation of a Low Coronal Shock Wave Observed in the EUV by the SDO/AIA. Astrophys. J. 738, 160. DOI ADS

Mann, G., Klassen, A., Aurass, H., Classen, H.T.: 2003, Development of shocks waves in the solar corona and the interplanetary space. In: Solar Wind Ten, American Institute of Physics Conference Series 679, 612. DOI ADS.

Mei, Z., Udo, Z., Lin, J.: 2012, Numerical experiments of disturbance to the solar atmosphere caused by eruptions. Science China Physics, Mechanics, and Astronomy 55, 1316. DOI] ADS

Moses, D., Clette, F., Delaboudinière, J.-P., Artzner, G.E., Bougnet, M., Brunaud, J., Carabetian, C., Gabriel, A.H., Hochedez, J.F., Millier, F., Song, X.Y., Au, B., Dere, K.P., 
Howard, R.A., Kreplin, R., Michels, D.J., Defise, J.M., Jamar, C., Rochus, P., Chauvineau, J.P., Marioge, J.P., Catura, R.C., Lemen, J.R., Shing, L., Stern, R.A., Gurman, J.B., Neupert, W.M., Newmark, J., Thompson, B., Maucherat, A., Portier-Fozzani, F., Berghmans, D., Cugnon, P., van Dessel, E.L., Gabryl, J.R.: 1997, EIT Observations of the Extreme Ultraviolet Sun. Solar Phys. 175, 571. DOI ADS

Muhr, N., Veronig, A.M., Kienreich, I.W., Temmer, M., Vršnak, B.: 2011, Analysis of Characteristic Parameters of Large-scale Coronal Waves Observed by the Solar-Terrestrial Relations Observatory/Extreme Ultraviolet Imager. Astrophys. J. 739, 89. DOI ADS

Muhr, N., Veronig, A.M., Kienreich, I.W., Vršnak, B., Temmer, M., Bein, B.M.: 2014, Statistical Analysis of Large-Scale EUV Waves Observed by STEREO/EUVI. Solar Phys. 289, 4563. DOI ADS

Newkirk, G. Jr.: 1961, The Solar Corona in Active Regions and the Thermal Origin of the Slowly Varying Component of Solar Radio Radiation. Astrophys. J. 133, 983. DOI. ADS.

Nitta, N.V., Schrijver, C.J., Title, A.M., Liu, W.: 2013, Large-scale Coronal Propagating Fronts in Solar Eruptions as Observed by the Atmospheric Imaging Assembly on Board the Solar Dynamics Observatory-an Ensemble Study. Astrophys. J. 776, 58. DOI ADS

Ouyang, Y., Zhou, Y.H., Chen, P.F., Fang, C.: 2017, Chirality and Magnetic Configurations of Solar Filaments. Astrophys. J. 835, 94. DOI ADS

Patsourakos, S., Vourlidas, A.: 2009, "Extreme Ultraviolet Waves" are Waves: First Quadrature Observations of an Extreme Ultraviolet Wave from STEREO. Astrophys. J. Lett. 700, L182. DOI ADS

Patsourakos, S., Vourlidas, A.: 2012, On the Nature and Genesis of EUV Waves: A Synthesis of Observations from SOHO, STEREO, SDO, and Hinode (Invited Review). Solar Phys. 281, 187. DOI ADS

Pesnell, W.D., Thompson, B.J., Chamberlin, P.C.: 2012, The Solar Dynamics Observatory (SDO). Solar Phys. 275, 3. DOI ADS

Schmidt, J.M., Ofman, L.: 2010, Global Simulation of an Extreme Ultraviolet Imaging Telescope Wave. Astrophys. J. 713, 1008. DOI ADS

Schmieder, B., Aulanier, G., Vršnak, B.: 2015, Flare-CME Models: An Observational Perspective (Invited Review). Solar Phys. 290, 3457. DOI

Sterling, A.C., Hudson, H.S.: 1997, Yohkoh SXT Observations of X-Ray "Dimming" Associated with a Halo Coronal Mass Ejection. Astrophys. J. Lett. 491, L55. DOI

Su, W., Cheng, X., Ding, M.D., Chen, P.F., Ning, Z.J., Ji, H.S.: 2016, Investigating the Conditions of the Formation of a Type II Radio Burst on 2014 January 8. Astrophys. J. 830, 70. DOI ADS.

Thompson, B.J., Myers, D.C.: 2009, A Catalog of Coronal "EIT Wave" Transients. Astrophys. J. 183, 225. DOI ADS

Thompson, B.J., Plunkett, S.P., Gurman, J.B., Newmark, J.S., St. Cyr, O.C., Michels, D.J.: 1998, SOHO/EIT observations of an Earth-directed coronal mass ejection on May 12, 1997. Geophys. Res. Lett. 25, 2465. DOI ADS.

Torrence, C., Compo, G.P.: 1998, A Practical Guide to Wavelet Analysis. Bulletin of the American Meteorological Society 79, 61. DOI

Vemareddy, P., Mishra, W.: 2015, A Full Study on the Sun-Earth Connection of an Earthdirected CME Magnetic Flux Rope. Astrophys. J. 814, 59. DOI ADS.

Veronig, A.M., Temmer, M., Vršnak, B.: 2008, High-Cadence Observations of a Global Coronal Wave by STEREO EUVI. Astrophys. J. Lett. 681, L113. DOI ADS

Wang, T., Yan, Y., Wang, J., Kurokawa, H., Shibata, K.: 2002, The Large-Scale Coronal Field Structure and Source Region Features for a Halo Coronal Mass Ejection. Astrophys. J. 572, 580. DOI ADS

Wang, Y.-M.: 2000, EIT Waves and Fast-Mode Propagation in the Solar Corona. Astrophys. J. Lett. 543, L89. DOI

Warmuth, A.: 2007, Large-scale Waves and Shocks in the Solar Corona. In: Lecture Notes in Physics, Berlin Springer Verlag, Lecture Notes in Physics, Berlin Springer Verlag 725, 107.

Warmuth, A.: 2010, Large-scale waves in the solar corona: The continuing debate. Advances in Space Research 45, 527. DOI ADS

Warmuth, A.: 2015, Large-scale Globally Propagating Coronal Waves. Living Reviews in Solar Physics 12. DOI ADS.

Webb, D.F., Lepping, R.P., Burlaga, L.F., DeForest, C.E., Larson, D.E., Martin, S.F., Plunkett, S.P., Rust, D.M.: 2000, The origin and development of the May 1997 magnetic cloud. J. Geophys. Res. 105, 27251. DOI ADS 
Wills-Davey, M.J., Attrill, G.D.R.: 2009, EIT Waves: A Changing Understanding over a Solar Cycle. Space Sci. Rev. 149, 325. DOI ADS

Wills-Davey, M.J., Thompson, B.J.: 1999, Observations of a Propagating Disturbance in TRACE. Solar Phys. 190, 467. DOI ADS.

Wu, S.T., Zheng, H., Wang, S., Thompson, B.J., Plunkett, S.P., Zhao, X.P., Dryer, M.: 2001, Three-dimensional numerical simulation of MHD waves observed by the Extreme Ultraviolet Imaging Telescope. J. Geophys. Res. 106, 25089. DOI ADS

Zheng, R., Chen, Y., Feng, S., Wang, B., Song, H.: 2018, An Extreme-ultraviolet Wave Generating Upward Secondary Waves in a Streamer-like Solar Structure. Astrophys. J. Lett. 858, L1. DOI. ADS.

Zhou, X.-p., Liang, H.-f.: 2017, A Study on the Fast Solar Corona Extreme-ultraviolet Wave Associated with a Coronal Mass Ejection. Chinese J. Astron. Astrophys. 41, 224. DOI ADS

Zhukov, A.N.: 2011, EIT wave observations and modeling in the STEREO era. Journal of Atmospheric and Solar-Terrestrial Physics 73, 1096. DOI ADS.

Zhukov, A.N., Auchère, F.: 2004, On the nature of EIT waves, EUV dimmings and their link to CMEs. Astron. Astrophys. 427, 705. DOI. ADS

Zong, W., Dai, Y.: 2017, Mode Conversion of a Solar Extreme-ultraviolet Wave over a Coronal Cavity. Astrophys. J. Lett. 834, L15. DOI. ADS

Zucca, P., Carley, E.P., Bloomfield, D.S., Gallagher, P.T.: 2014, The formation heights of coronal shocks from 2D density and Alfvén speed maps. Astron. Astrophys. 564, A47. DOI. ADS 

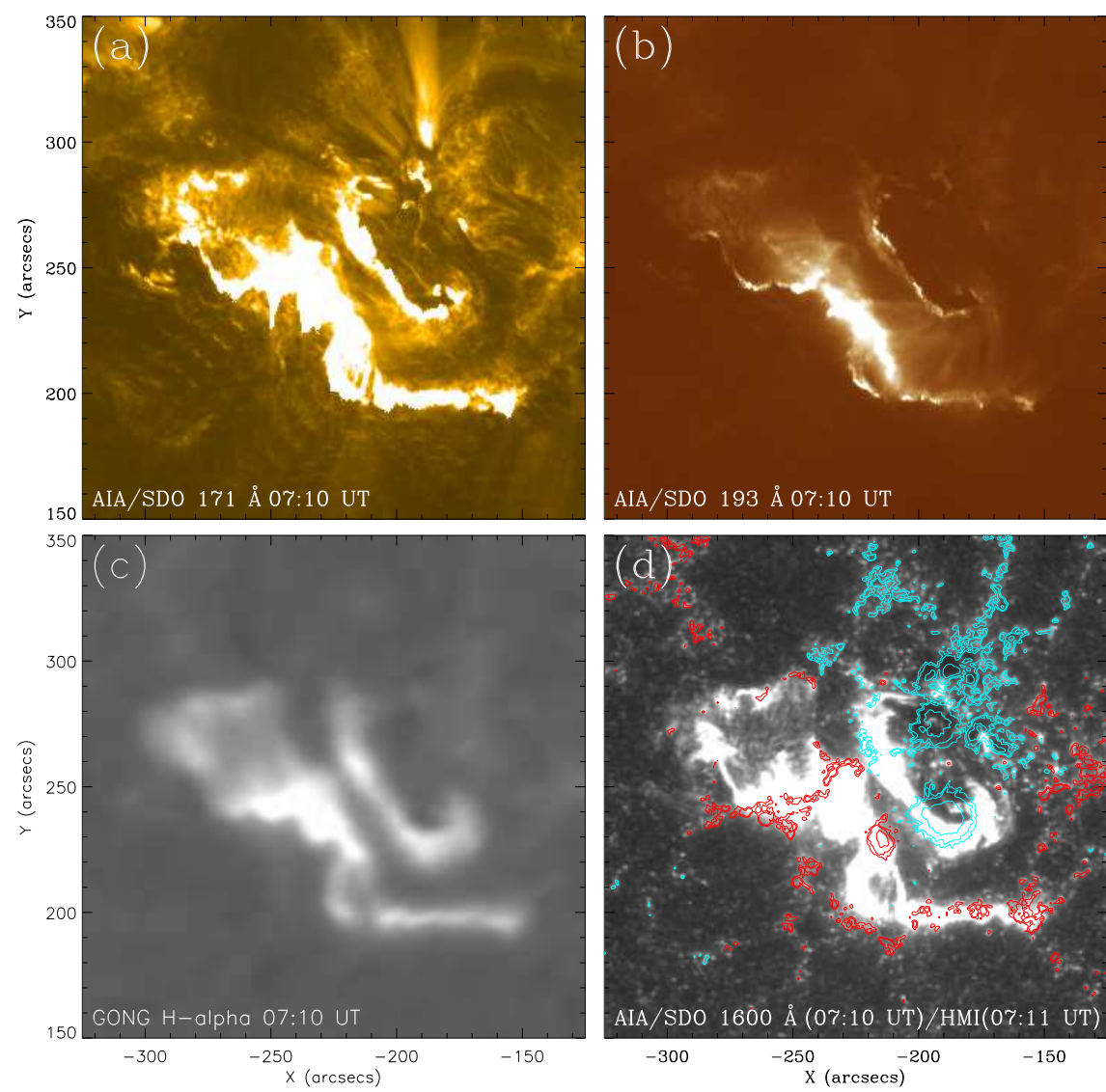

Figure 1. Multiwavelength observations of the flare ribbons in AIA $171,193,1600 \AA$ and $\mathrm{H} \alpha$ at 07:10 UT where the AIA $1600 \AA$ image is overlaid by HMI contours (d). Red/cyan colors represent negative/positive magnetic polarities respectively. The contour levels are $\pm 200, \pm 400$, $\pm 800, \pm 1600 \mathrm{G}$. 

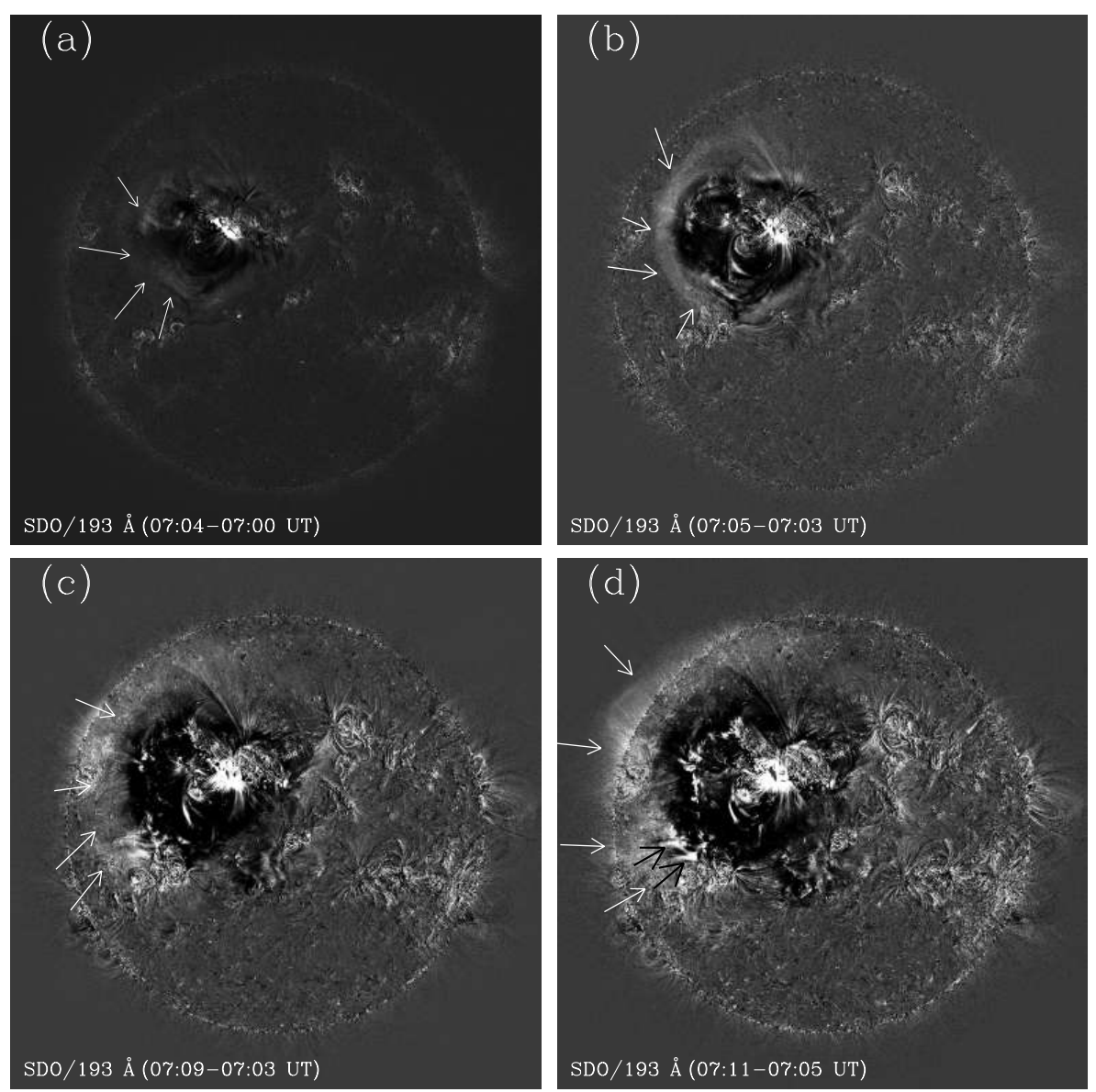

Figure 2. Temporal and spatial evolution of the EUV wave in full disk running difference images at AIA $193 \AA$ A. Arrows indicate the location of the propagating bright fronts. The white arrows indicate the fast-mode MHD wave and the black arrows indicate the slow-component EUV wave. 

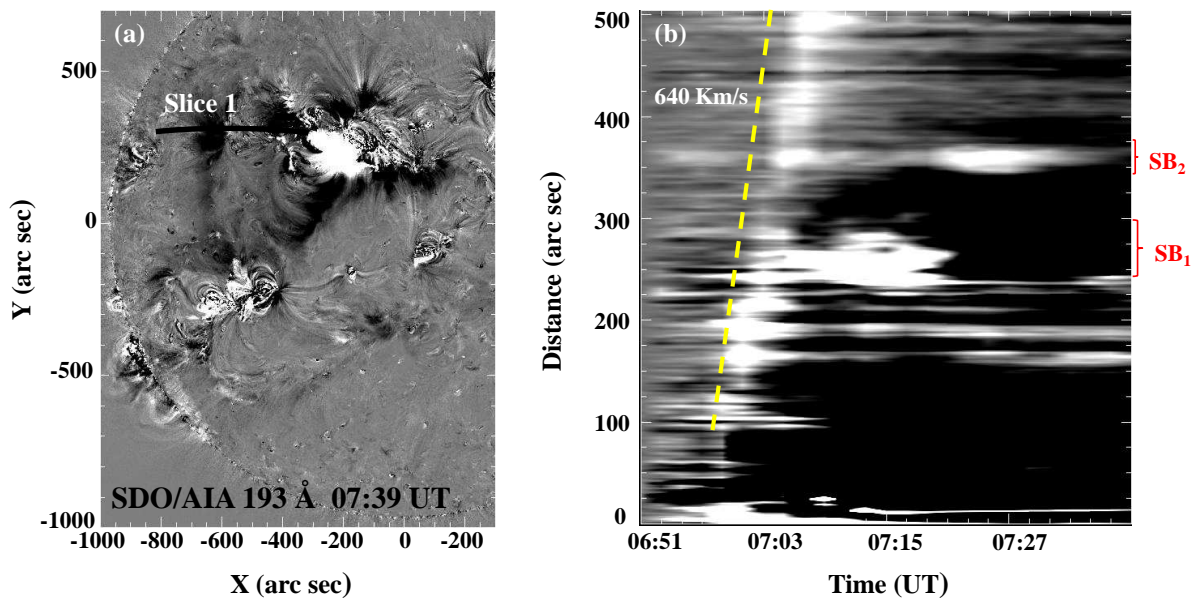

Figure 3. (a): SDO/AIA $193 \AA$ difference image at 07:39 UT showing the direction of slice 1 (black line) used for the time-distance diagram in the right panel. (b): Time-distance diagram showing a fast-component EUV wave and two resulting stationary brightenings $S B_{1}$ and $S B_{2}$. 


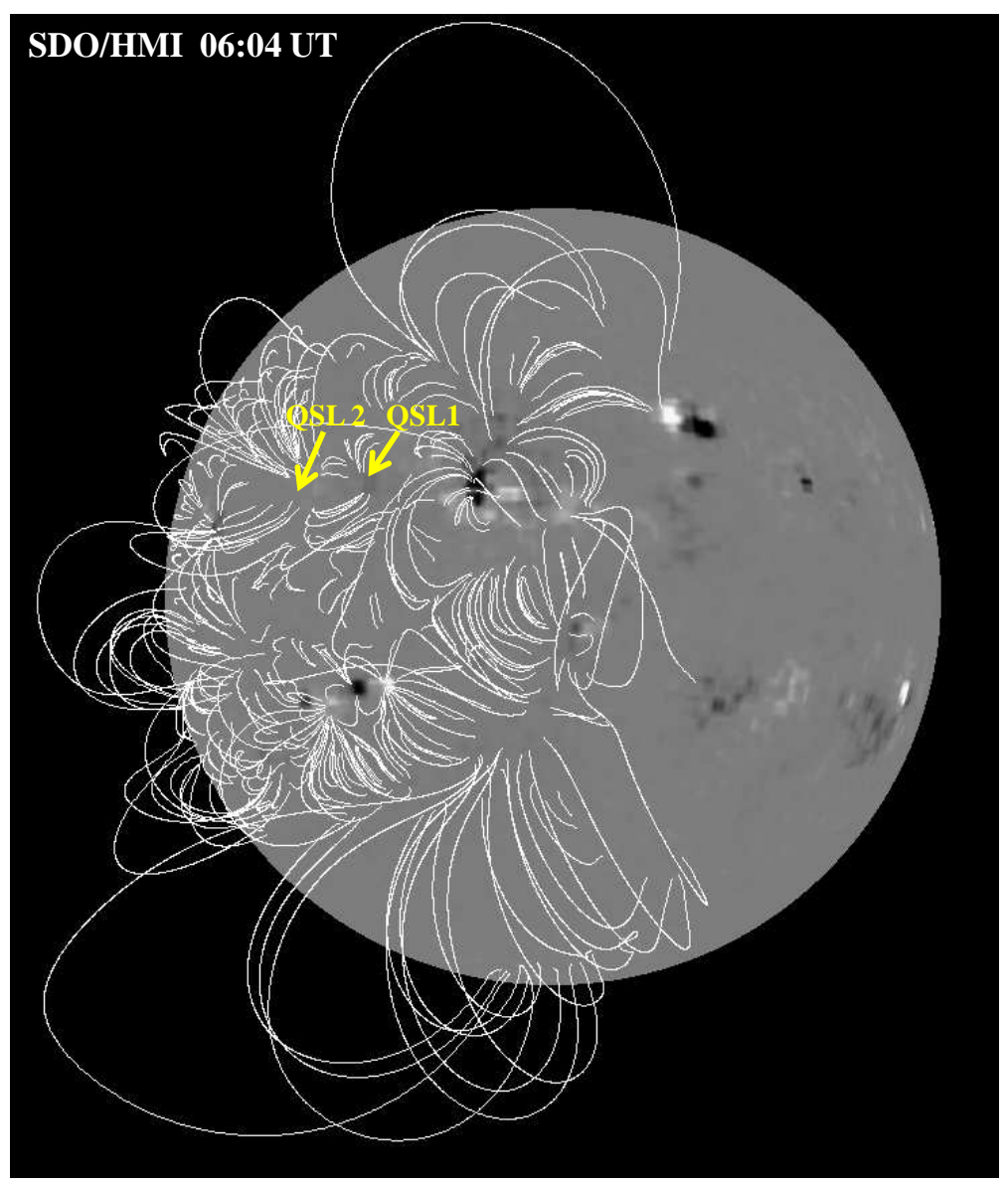

Figure 4. Potential coronal magnetic field extrapolated from the HMI magnetic field at 06:04 UT. Yellow arrows indicate the locations of QSLs. 

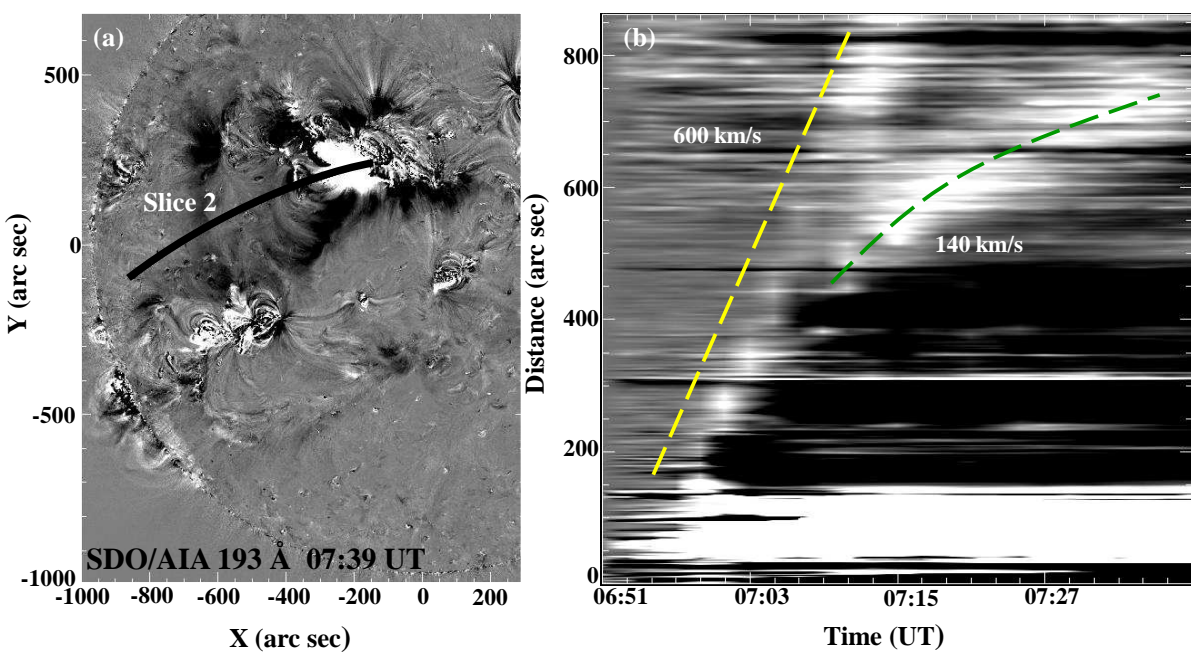

Figure 5. (a): SDO/AIA $193 \AA$ Åfference image at 07:39 UT showing the direction of slice 2 (black line) used for the time-distance diagram in the right panel. (b): Time-distance diagram showing both the fast- and slow-component EUV waves. 


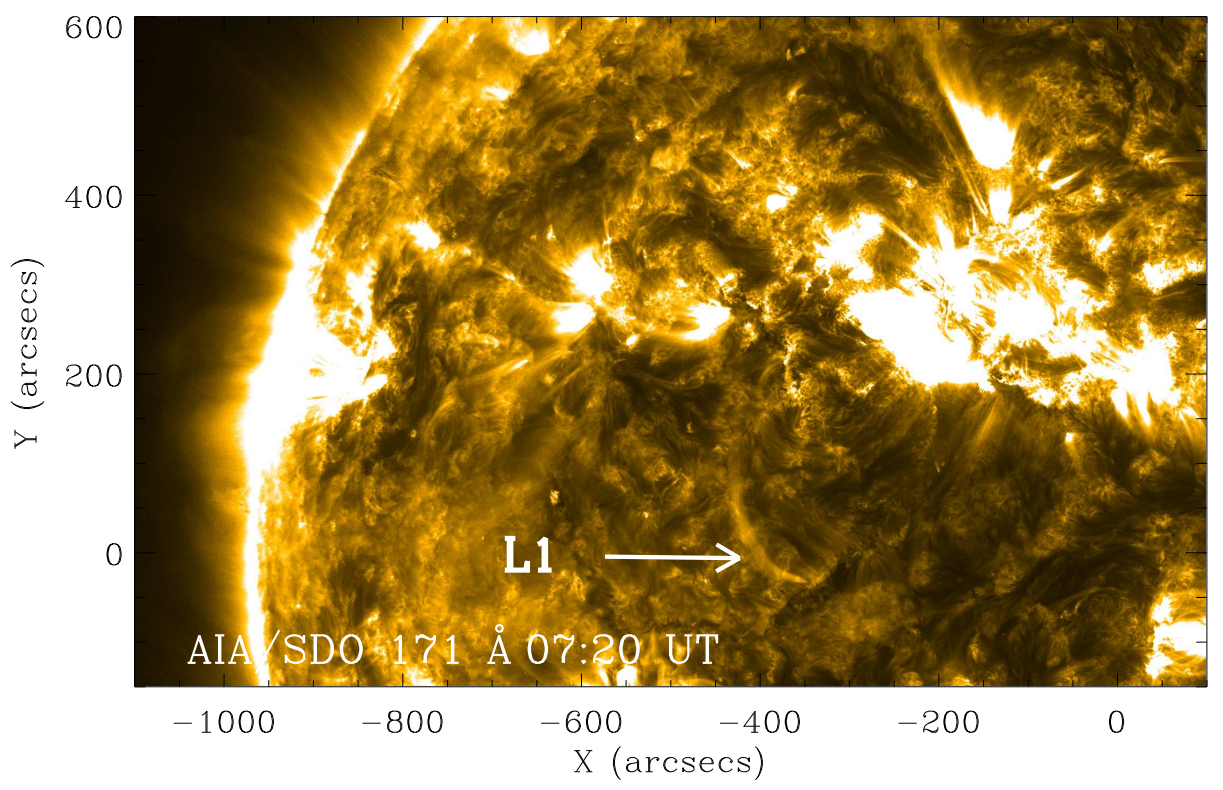

Figure 6. AIA/SDO $171 \AA$ image showing the oscillating coronal loop $L_{1}$ near the flare site. 
(a)

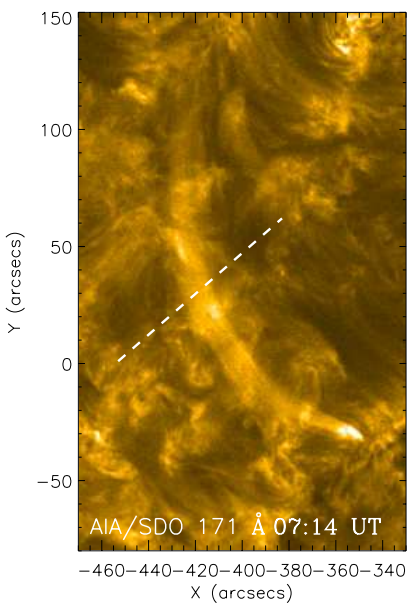

(b)

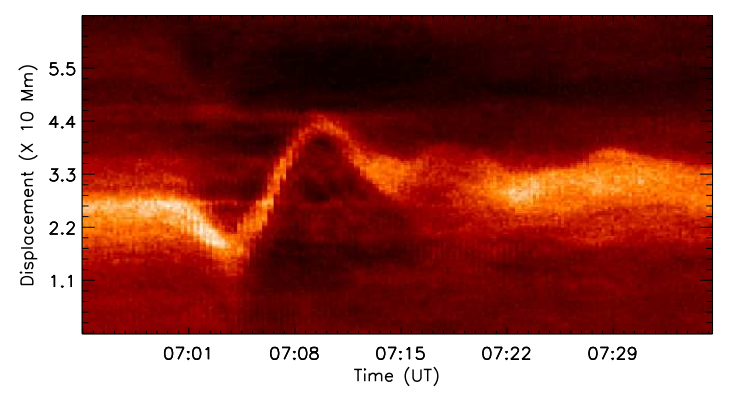

Figure 7. (a): Oscillating loop in AIA/SDO $171 \AA$. The location of the artificial slit used for the timeslice analysis is shown by the white dashed line. (b): The time-distance plot of the oscillations of the loop along the slit shown in the left panel. 


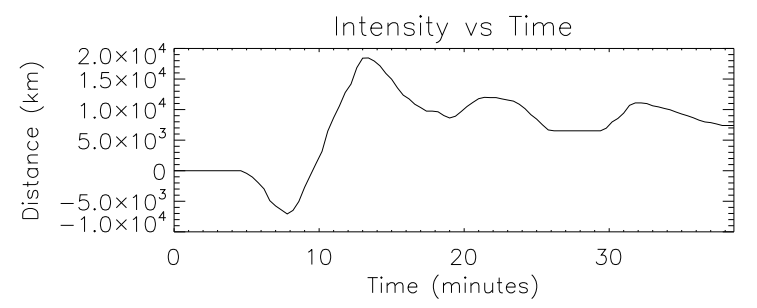

Global Period at max. power (<845.9 sec.) $=541.37 \mathrm{sec}$

Prob. level: $99-100 \%$
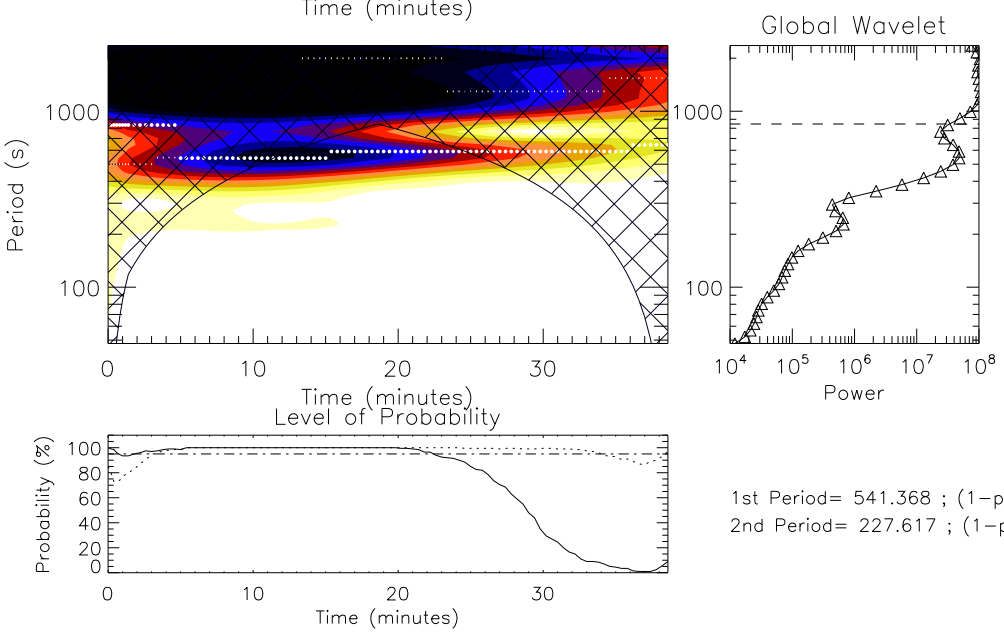

1 st Period $=541.368 ;(1-p)=1.00000$ 2nd Period $=227.617 ;(1-p)=1.00000$

Figure 8. The wavelet result for the loop oscillations in AIA $171 \AA$. The top panel shows the intensity variation with time in AIA $171 \AA$. The wavelet power spectrum is shown in the middle panel, and the probability is given in the bottom panel. 

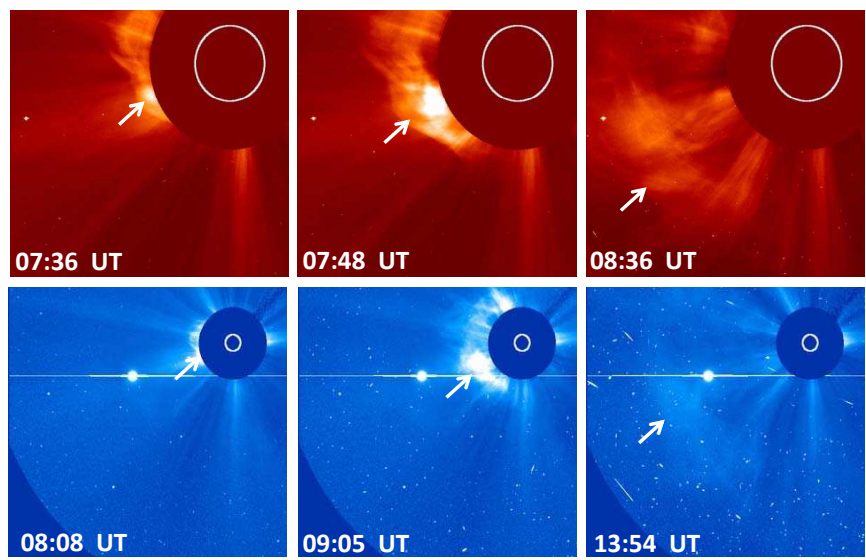

Figure 9. Evolution of the associated CME observed by the LASCO C2 (top) and C3 (bottom) coronagraphs.

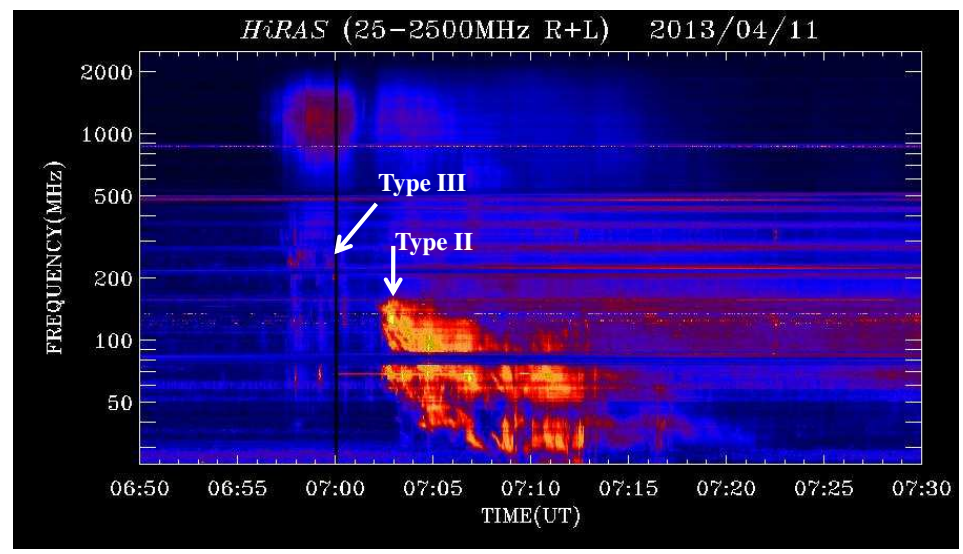

Figure 10. The dynamic spectrum observed by the Hiraiso Radio Spectrograph (HiRAS) in 25-2500 MHz on 2013 April 11 showing type II and type III radio bursts during the flare/CME event. 


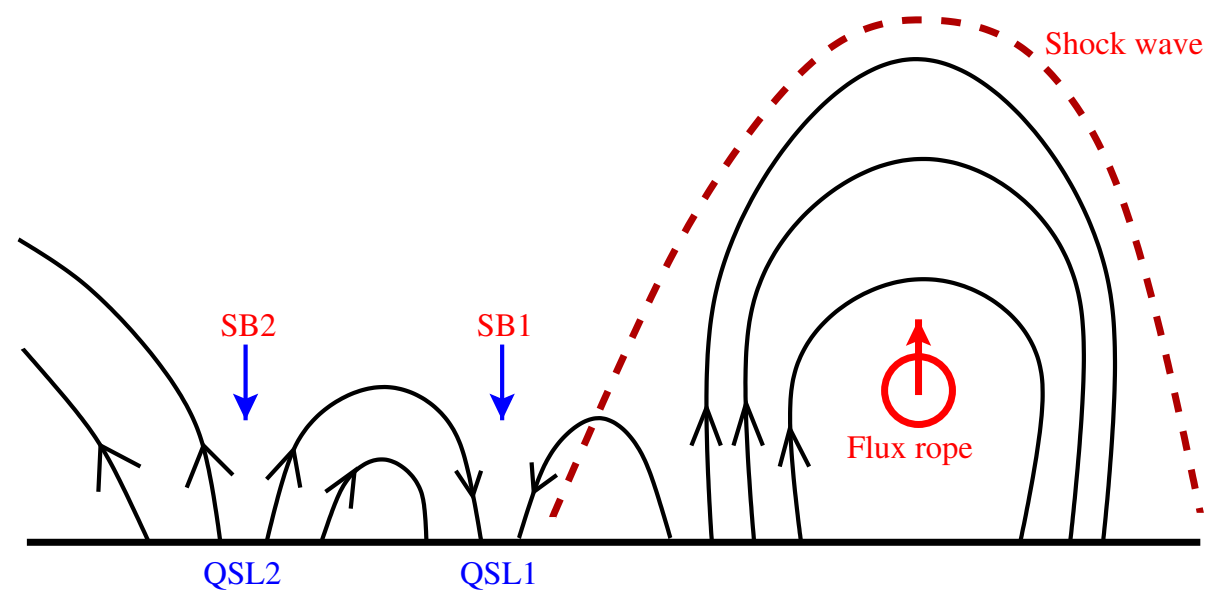

Figure 11. Cartoon showing the propagation of the fast mode EUV wave/shock wave (red dashed arc). The black solid lines represent the initial magnetic configuration. The fast-mode EUV wave encounters two QSLs, producing two stationary brightenings. 